might, for example, improve how materials transport spin currents. There is an overlap in potential applications with a topological phenomenon called the quantum anomalous Hall effect (QAHE), which is generated using magnetic impurities in thin films of topological insulators ${ }^{10,11}$. Steps on the surface of an antiferromagnetic topological insulator produce the same perfectly conducting edge channels as in the QAHE.

The fact that manganese bismuth telluride is intrinsically magnetic, rather than having its magnetism result from randomly located impurities, as in current QAHE materials, could be advantageous. Key questions remain about how the magnetism varies between different samples of this material. In particular, it seems that both the magnetic transition temperature and the nature of the magnetic ordering between planes could vary, and that an applied magnetic field might be used to switch this ordering. At a more fundamental level, antiferromagnetic topological insulators are predicted to support, without applied electric or magnetic fields, a quantized electromagnetic response (one that comes in discrete units), known as axion electrodynamics ${ }^{6}$. The current papers show how the synthesis and theory of crystals that have various symmetries combine to reveal important types of electronic order.

\section{Roger S. K. Mong is in the Department} of Physics and Astronomy, University of Pittsburgh, Pittsburgh, Pennsylvania 15620, USA. Joel E. Moore is in the Department of Physics, University of California, Berkeley, Berkeley, California 94720, USA, and at the Materials Sciences Division, Lawrence Berkeley National Laboratory, Berkeley, California.

e-mails: rmong@pitt.edu; jemoore@berkeley. edu

\footnotetext{
1. Otrokov, M. M. et al. Nature 576, 416-422 (2019).

2. Rienks, E. D. L. et al. Nature 576, 423-428 (2019).

3. Hasan, M. Z. \& Kane, C. L. Rev. Mod. Phys. 82, 3045-3067 (2010).

4. Moore, J. E. Nature 464, 194-198 (2010).

5. Fu, L. \& Kane, C. L. Phys. Rev. Lett. 100, 096407 (2008).

6. Mong, R. S. K., Essin, A. M. \& Moore, J. E. Phys. Rev. B 81, 245209 (2010)

7. Chen, Y. L. et al. Science 325, 178-181 (2009).

8. Gong, Y. et al. Chinese Phys. Lett. 36, 076801 (2019).

9. Huang, B. et al. Nature 546, 270-273 (2017).

10. Chang, C.-Z. et al. Science 340, 167-170 (2013).

11. Tokura, Y., Yasuda, K. \& Tsukazaki, A. Nature Rev. Phys. 1, 126-143 (2019).
}

\title{
Antitumour T cells stand the test of time
}

\section{Miguel Reina-Campos \& Ananda W. Goldrath}

Enhancing antitumour immune responses has revolutionized

cancer treatment, yet some hurdles impede this approach. The discovery of a way to boost the lifespan and function of antitumour immune cells removes a key obstacle. See p.471

Immune cells called cytotoxic CD8 T cells can directly kill tumours and are key weapons mobilized in many immunotherapy approaches used in the clinic. However, the cells' activity can be thwarted by the ability of tumours to create harsh microenvironments, recruit immunoregulatory cells and induce inhibitory signals that hamper T-cell function, accumulation and tumour infiltration. On page 471 , Wei et al. ${ }^{1}$ report that depletion of the protein REGNASE-1 extends the survival of antitumour CD8 T cells and enhances their function, enabling the cells to fight cancer more effectively.

The development of anticancer clinical strategies that use immune cells has profoundly improved the treatment of certain malignancies. The delivery of $\mathrm{T}$ cells that can specifically target tumours is used in an approach called adoptive T-cell therapy (ACT), which relies on T cells that have been taken from a person's blood or tumour. These cells are stimulated in the laboratory to cause them to divide and to increase the number of

\section{"This study offers a strong incentive to investigate the use of combinatorial approaches."}

antitumour $\mathrm{T}$ cells, and, in some cases, they are modified to enhance their ability to eliminate cancer cells ${ }^{2}$. For example, $\mathrm{T}$ cells can be engineered to express a receptor, called a chimaeric antigen receptor (CAR-T), that specifically targets tumours, and such cells are remarkably successful at treating leukaemia.

Despite the immense potential of this strategy, the use of ACT is currently limited because the modified $\mathrm{T}$ cells that are transferred back to a person with cancer can be short-lived, and are often unable to overcome a tumour's ability to hinder their function. When naive $T$ cells recognize a disease-causing agent or a tumour cell, they proliferate to form shortlived tumour-killing (that is, cytotoxic) CD8 T cells (also known as effector cells) that kill these infected or malignant cells (Fig. 1). If the infection or the tumour cells are eliminated, most of these CD8 T cells die, but a small population remains in the form of long-lived memory $T$ cells, which are self-renewing and can generate cytotoxic CD8 T cells if the same infection or malignancy is encountered again ${ }^{3}$.

However, if the infection or tumour cannot be eliminated, the cytotoxic T cells progressively lose their function (a process termed exhaustion). The ideal population of T cells for use in ACT would infiltrate tumours and accumulate in substantial numbers while retaining cytotoxic function and the capacity for self-renewal ${ }^{2}$. Yet the differentiation of T cells into cytotoxic CD8 T cells impairs successful retention of the potential to form longlived memory cells. This raises the question of whether a strategy can be found to induce both of these beneficial traits in T cells used for ACT. It has been speculated that, in the unforgiving tumour microenvironment, CD8 T cells would need to have a robust metabolism to sustain the nutritional and energetic requirements needed for survival and to retain their antitumour activity ${ }^{4}$.

Wei et al.used the CRISPR-Cas9gene-editing technology to disrupt more than 3,000 genes associated with metabolism in T cells, to test their functions in a mouse model of antitumour ACT. The authors identified more than 200 genes that have a striking ability to affect the persistence and function of the CD8 T cells transferred into tumour-bearing mice. The disruption of many genes had a negative effect on the ability of the cells to persist and thus accumulate in tumours, but the disruption of four genes resulted in a much higher than normal number of T cells infiltrating the tumours.

At the top of this list is the gene that encodes the enzyme REGNASE-1. Its deletion in CD8 T cells caused 2,000 times more of these cells to accumulate in tumours than did CD8 T cells that expressed REGNASE-1. This enzyme binds to and degrades RNA, and influences immune responses ${ }^{5-7}$, but its role in the antitumourfunction of CD8T cellshad notbeen explored. CD8 T cells that lacked REGNASE-1 were better than wild-type CD8 T cells at fighting two types of tumour in mice: an aggressive skin cancer called melanoma and a blood cancer termed acute lymphocytic leukaemia. The REGNASE-1-deficient CD8 $\mathrm{T}$ cells proliferated at a similar rate to 


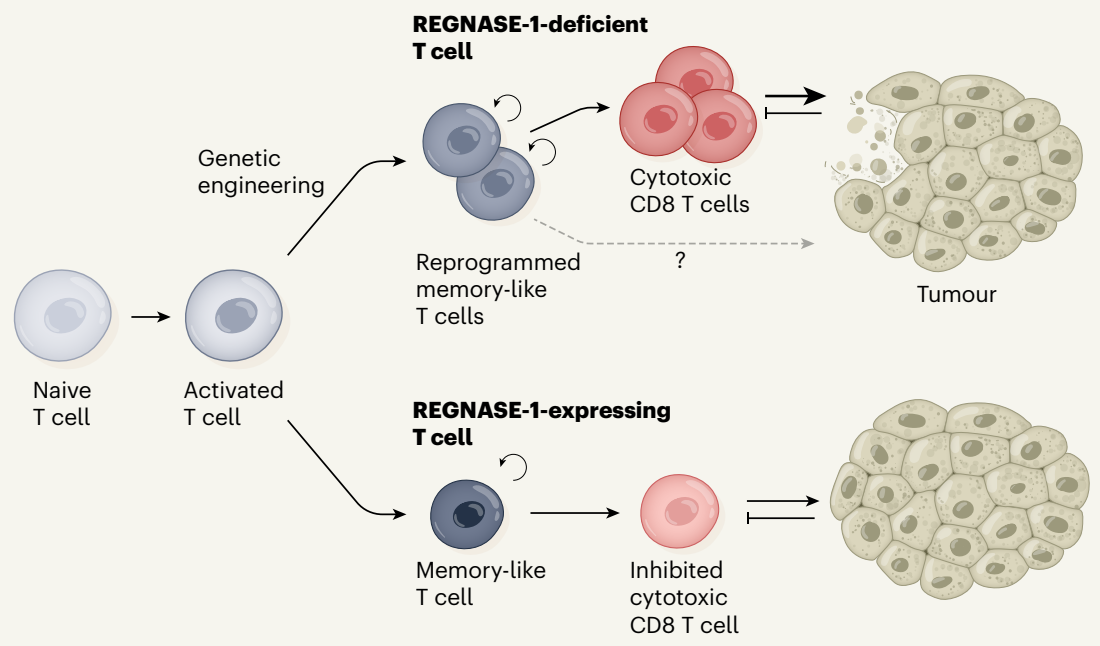

Figure 1 | Boosting $T$ cells to enable a sustained antitumour response. Immunotherapy uses immune cells called T cells to target cancer in the clinic; however, these tumour-killing cytotoxic CD8 T cells are shortlived. Moreover, they can be inhibited by the tumour microenvironment and lose their functional activity because of chronic stimulation if the tumour is not eliminated. Wei et al. ${ }^{1}$ studied the factors affecting T-cell responses against tumours in mouse models. A T-cell response begins when a naive T cell becomes activated by recognizing its target protein (not shown). Activated T cells can give rise to memory-like T cells that can self renew or generate cytotoxic CD8 T cells. The authors report that if $\mathrm{T}$ cells are engineered to lack the protein REGNASE-1, they are reprogrammed to form high numbers of memory-like T cells and have enhanced antitumour activity compared to the case of REGNASE-1-expressing T cells. Therefore, if T cells are REGNASE-1 deficient, they are better equipped to sustain high numbers of cytotoxic CD8 T cells in tumours and produce a prolonged antitumour response. Loss of REGNASE-1 provides the joint benefit of generating T cells that have more tumour-killing activity and memory-like qualities. Whether these reprogrammed memory-like T cells directly contribute to tumour-killing activity is unknown. By contrast, T cells that express REGNASE-1 produce a short-lived antitumour response that fails to overcome tumour inhibition of the immune response.

the wild-type cells, but did not die as rapidly, allowing them to accumulate.

To better understand how REGNASE-1 deficiency resulted in this increased persistence of $\mathrm{T}$ cells, the authors analysed geneexpression profiles of REGNASE-1-deficient and wild-type cells. REGNASE-1 deficiency was linked with an increase in a molecular signature characteristic of memory T cells, suggesting the presence of a larger than normal population of long-lived memory-like cells that can give rise to cytotoxic CD8 T cells. REGNASE-1deficient CD8 T cells showed striking increases in mitochondrial function (mitochondria are organelles that provide a crucial source of cellular energy), including the ability to produce energy and consume oxygen. This is notable because this capacity is often compromised in tumour-fighting T cells ${ }^{8}$. These combined effects of REGNASE-1 deficiency enabled CD8 T cells and CAR-T cells used for ACT to accumulate and remain active over time in the cancers targeted in the mouse models.

To further understand this mechanism, Wei and colleagues used CRISPR-Cas9 to disrupt approximately 20,000 genes in REGNASE-1-deficient CD8T cells, to pinpoint key downstream genes that mediate the REGNASE-1-dependent cellular reprogramming. The inactivation of the transcription factor BATF, a key regulator of the differentiation of CD8 T cells ${ }^{9}$, abolished the long lifespan of T cells lacking REGNASE-1 and their high expression of genes associated with mitochondria. The authors found that the combined depletion of REGNASE-1 with that of either of the proteins PTPN2 or SOCS1 had a synergistic effect that increased the persistence, accumulation and antitumour activity of $\mathrm{T}$ cells compared with the properties of $T$ cells that were deficient only in REGNASE-1.

Wei and colleagues report that REGNASE-1-deficient CD8 T cells had a higher expression of cytotoxic proteins than did wild-type CD8 T cells in both the memory-like and the cytotoxic CD8 T cells in tumours. Wild-type cells with memory-like properties typically do not kill tumour cells directly ${ }^{10}$. It is not clear whether REGNASE-1-deficient memory-like $T$ cells function solely to self-renew and produce the cytotoxic CD8-T-cell population, or whether they can also directly mediate tumour-cell killing, given that their expression of cytotoxic molecules is higher than that of wild-type CD8 T cells.

If they do have a role in tumour-cell killing, how do these REGNASE-1-deficient memory-like CD8 T cells manage to both do this and maintain the population of cytotoxic CD8 T cells? Intriguingly, the authors show that, for increased persistence, the
REGNASE-1-deficient CD8 T cells need to encounter the tumour protein that they recognize. This might explain why the accumulation of antitumour $\mathrm{T}$ cells was more pronounced in mouse tumours than in the animals' spleens, which are rich in T cells but are located away from the sites of exposure to the tumour proteins.

It remains to be investigated whether other cues in the tumour microenvironment contribute to boosting the persistence of REGNASE-1-deficient CD8 T cells. To this end, it might be informative to assess the metabolic profile of CD8 T cells in the tumour that have combinatorial depletions of REGNASE-1, PTPN2, SOCS1 and BATF. This could provide insights into the effect of these proteins on the reprogramming of CD8-T-cell metabolism and to what extent this is important for the cells' differentiation and antitumour function. Also, finding the relevant metabolites in this context might provide clues to how these CD8 T cells can be influenced by a nutritionally depleted tumour microenvironment.

Wei and colleagues' study reveals promising leads that might result in advances in ACT-based immunotherapies. It will be worth testing whether engineering CD8 T cells to delete or express low levels of the gene encoding REGNASE-1 would be feasible as part of the manufacturing process for CAR-T cells. Finally, given that the inhibition of PTPN2 in tumour cells sensitizes them to immunotherapy ${ }^{11}$, this study offers a strong incentive to investigate the use of combinatorial approaches, including REGNASE-1 and PTPN2 inhibitors, as a way to reprogram CD8 T cells to improve current therapies.

\section{Miguel Reina-Campos and Ananda W.}

Goldrath are in the Division of Biological Sciences, University of California, San Diego, La Jolla, California 92093, USA. e-mails: agoldrath@ucsd.edu; mreinacampos@ucsd.edu
1. Wei, J. et al. Nature 576, 471-476 (2019). 2. Lim, W. A. \& June, C. H. Cell 168, 724-740 (2017).

3. Henning, A. N., Roychoudhuri, R. \& Restifo, N. P. Nature Rev. Immunol. 18, 340-356 (2018).

4. Zhang, L. \& Romero, P. Trends Mol. Med. 24, 30-48 (2018).

5. Matsushita, K. et al. Nature 458, 1185-1190 (2009).

6. Iwasaki, H. et al. Nature Immunol. 12, 1167-1175 (2011).

7. Uehata, T. et al. Cell 153, 1036-1049 (2013).

Scharping, N. E. et al. Immunity 45, 701-703 (2016).

Kurachi, M. et al. Nature Immunol. 15, 373-383 (2014).

10. Miller, B. C. et al. Nature Immunol. 20, 326-336 (2019).

11. Manguso, R. T. et al. Nature 547, 413-418 (2017). go.nature.com/2lpijge for details.

This article was published online on 11 December 2019. 